\title{
Clinical Implication of Facial Nerve Decompression in Complete Bell's Palsy: A Systematic Review and Meta-Analysis
}

\author{
Sang-Yeon Lee ${ }^{1}$ (i) $\cdot$ Jeon Seong ${ }^{2}$ (D) $\cdot$ Young Ho Kim² (i) \\ ${ }^{l}$ Department of Otorhinolaryngology-Head and Neck Surgery, Seoul National University Hospital, Seoul National University College of Medicine, \\ Seoul; ' Department of Otorhinolaryngology-Head and Neck Surgery, SMG-SNU Boramae Medical Center, Seoul National University College of \\ Medicine, Seoul, Korea
}

We compared the therapeutic efficacy of facial nerve decompression (FND) and conservative treatment in patients with Bell's palsy through a systematic review and meta-analysis. Primary database search was performed in PubMed, Medline, and Embase. After screening, 13 studies were assessed for their eligibility. Among them, seven studies employing either the House-Brackmann grading system (HBGS) or May's classification (modified HBGS) were selected for quantitative and qualitative analysis. Based on May's classification, the degree of recovery was classified into complete (HBGS I), fair (HBGS II-III), or failed (HBGS IV-VI) recovery. The outcomes were assessed between 6 and 12 months after surgery. The estimated pooled odds ratio (OR) and $95 \%$ confidence interval $(\mathrm{CI})$ were calculated using random effects model. Cohorts were comprised of patients who underwent FND $(n=202,53.0 \%)$ and conservative treatments $(n=179,47.0 \%)$. In pooled analysis, the rate of complete recovery was significantly higher in the FND group than in the control group (OR, 2.06; 95\% CI, 1.22 to $3.48 ; P=0.007)$ showing neither heterogeneity nor publication bias. Meanwhile, the rates of fair recovery (OR, 0.71 ; $95 \% \mathrm{CI}, 0.42$ to $1.21 ; P=0.208)$ and failed recovery (OR, $0.60 ; 95 \% \mathrm{CI}, 0.22$ to $1.67 ; P=0.327)$ in the FND group were similar to that in the control group. In subgroup analyses, there was no significant difference in the OR according to the operation timing and surgical approach. FND can be a possible treatment option for patients with complete Bell's palsy, especially for complete recovery, which provide insights on decision-making and outcome prediction. However, FND should be determined carefully given the risk of small study effects and possible complications.

Keywords. Bell Palsy; Facial Nerve Decompression; Meta-Analysis

\section{INTRODUCTION}

Facial nerve palsy (FNP) is typically a paresis or paralysis of the unilateral facial muscles. Idiopathic FNP, also known as Bell's palsy, accounts for approximately $75 \%$ of acute FNP cases [1]. Although most patients with Bell's palsy recover from the func-

\footnotetext{
- Received April 9, 2019

Revised July 12, 2019

Accepted July 15, 2019

- Corresponding author: Young Ho Kim

Department of Otorhinolaryngology-Head and Neck Surgery, SMG-SNU Boramae Medical Center, Seoul National University College of Medicine, 20 Boramae-ro 5-gil, Dongjak-gu, Seoul 07061, Korea

Tel: +82-2-870-2442, Fax: +82-2-870-3863

E-mail: yhkiment@gmail.com
}

tional nerve dysfunction following the initial insult [2], 30\% of complete facial palsy patients exhibited an incomplete recovery that was closely linked to aesthetic, psychologic, and social problems [3]. In addition, complete facial palsy detected during early evaluation is known to be associated with poor prognosis [4].Therefore, understanding of nature prognosis and timely intervention are vital to achieving optimal therapeutic outcomes, especially in the complete type of Bell's palsy.

High-dose systemic corticosteroid administration is the key to the initial treatment of Bell's palsy [5]. Antiviral agents may be implicated in combination with steroids to restore the facial nerve function, even though evidence on the effectiveness of combination therapy is still controversial [6]. Nonetheless, previous a large cohort study demonstrated some extent of patients

Copyright @ 2019 by Korean Society of Otorhinolaryngology-Head and Neck Surgery.

This is an open-access article distributed under the terms of the Creative Commons Attribution Non-Commercial License (http://creativecommons.org/licenses/by-nc/4.0)

which permits unrestricted non-commercial use, distribution, and reproduction in any medium, provided the original work is properly cited. 
showed incomplete recovery or no recovery at all, despite combination therapy with prednisolone and valganciclovir [7]. In cases involving severe nerve degeneration within 14 days after the onset of complete facial palsy, facial nerve decompression (FND) can be considered as a possible surgical option to restore facial function [3]. The outcome of treatment with FND has been shown to be variable and depends on the timing of the treatment, approach of surgery, or other accompanying factors of FNP $[8,9]$.

A previous Cochrane review on early surgical interventions for Bell's palsy reported that there was an insufficient evidence to demonstrate that FND was beneficial [10]. However, the aforementioned Cochrane review was based only on two studies which involved FND using a transmastoid approach. Since the publication of this Cochrane review, studies aiming to compare FND and medical treatment have emerged. Nonetheless, a recent meta-analysis showed that FND does not lead to significant improvement in facial nerve function compared to medical treatment based on the House-Brackmann grading system (HBGS) [11]. However, the results were limited by a significant higher heterogeneity across the studies $\left(I^{2}=89.72\right)$. Moreover, the bias related to small-study effects, such as publication bias, was not thoroughly addressed in that study. Thus, the therapeutic role of FND in the treatment of complete Bell's palsy needs to be re-established.

We, herein, aimed to compare therapeutic efficacy between FND and conservative treatments in patients with complete Bell's palsy through a systematic review and meta-analysis. Additionally, we performed subgroup analyses according to the timing of treatment and approach of surgery with meticulous interpretations of bias.

\section{MATERIALS AND METHODS}

This systematic review was conducted using the Preferred Re-

\section{H I G H L I G H T S}

- In patients with complete Bell's palsy, facial nerve decompression (FND) leads to a higher complete recovery rate compared to conservative treatment.

- There was no significant difference in the odds ratio for fair and failed recovery.

- In subgroup analyses according to operation timing and surgical approach, no significant difference of facial function recovery was found.

- FND can be a possible treatment option for patients with complete Bell's palsy, especially for complete recovery.

- FND should be determined carefully when considering the risk of bias and possible complications. porting Items for Systematic Reviews and Meta-Analyses (PRISMA) checklists. A PRISMA flow diagram was used to describe the flow of information during various phases of the systematic review [12]. This study utilized quantitative methods to examine the reasons for variation in treatment outcomes.

\section{Search strategy}

Two of the authors (SYL and YHK) independently searched PubMed, Medline, and Embase databases. Available studies in the databases from their inception to June 30, 2018 were searched. We employed the following search syntax, adapted to each database as appropriate: \#1 (Bell's palsy) OR (Bell palsy) OR (idiopathic facial paralysis) OR (idiopathic facial palsy) OR (facial paralysis) OR (facial palsy) AND \#2 (decompression) OR (facial nerve decompression) OR (facial decompression) OR (transmastoid decompression) OR (middle fossa decompression).

\section{Selection of studies}

Two authors (SYL and YHK) reviewed all retrieved articles by screening the titles and abstracts. Full texts of eligible articles were subsequently evaluated to determine whether they met the inclusion criteria. Only studies which involved the following were included in the analysis: (1) participants who presented with idiopathic facial palsy (Bell's palsy); (2) direct comparison of recovery of facial nerve function between FND and conservative treatments; and (3) the measurements of outcomes using HBGS or modified HBGS (May's classification). Indeed, FND in the included studies was performed in patients with persistent facial palsy following medical treatment, except for the one study. Brown [13] designed a double-blind study to test the facial nerve function; thereby, 41 cases received only steroid therapy while 41 cases underwent FND without preceding medical treatment. The medical treatment includes the steroid and/or antiviral therapy, regardless of different treatment regimen in perspectives of dose and duration. The conservative treatment refers to cases of the medical administration as the same protocol of FND group followed by observation. The following publications were excluded: (1) publications which involved patients diagnosed with herpes zoster, who had facial palsy with traumatic etiology or recurrent facial palsy; (2) review articles and case reports; and (3) publications with inaccessible original articles (e.g., only abstracts were available) and/or with incomplete data; and (4) duplicate publications.

\section{Data extraction}

Two authors independently extracted data; any discrepancies were resolved by consensus between the two authors. For the meta-analysis, the following information was obtained: author, year of publication, study design, number of participants, inclusion criteria, surgical approach, treatment outcomes, evaluation period, and complications. 


\section{Outcomes criteria}

We mainly focused on the degree of improvement in facial nerve function after FND compared to conservative treatment. Based on May's evaluation method (modified HB grading), the degree of facial nerve function was classified into complete recovery (grade HB I), fair recovery (grade HB II, III), and failed recovery (grades HB IV,V, and VI) (Supplementary Table 1). The treatment outcomes between 6 months and 1 year after treatment were evaluated in this study. There were insufficient studies to enable any statistical analysis of continuous data, such as changes in electroneurography and electromyography scores. Furthermore, subgroup analyses according to surgical approach and operation timing were assessed among available studies. Specifically, operation timing was classified into early ( $<14$ days) and delayed ( $\geq 14$ days) interventions. Additionally, we analyzed the complications after FND, when available.

\section{Data synthesis and measurement of treatment outcomes}

In this study, the estimated pooled odds ratios (ORs) and 95\% confidence intervals (CIs) were calculated using the random effects model. Selection of random effects was determined based on a conceptual understanding of the presence of population effects within enrolled studies, rather than using the statistical results of homogeneity tests. Analysis of pooled proportions was performed; cases with missing or incomplete information were excluded. For dichotomous outcome data (May's classification), the pooled ORs and their $95 \%$ CIs were calculated. For continuous outcome data (HBGS), the standardized mean differences (SMDs) with 95\% CIs were measured. All analyses were performed using the R software package ver. 3.3.2 (R Foundation for Statistical Computing, Vienna, Austria).

\section{Assessment of heterogeneity and interpretation}

We calculated the $\mathrm{I}^{2}$ statistic to evaluate rates of heterogeneity across the studies. If an $\mathrm{I}^{2}$ value of $>50 \%$ and a $P$-value of

\begin{tabular}{|c|}
\hline $\begin{array}{c}\text { Comparison of therapeutic effects between facial nerve decompression and conservative treatment } \\
\text { for idiopathic facial nerve palsy (Bell's palsy) } \\
\text { (inception date: Aug 21, 2018) }\end{array}$ \\
\hline
\end{tabular}
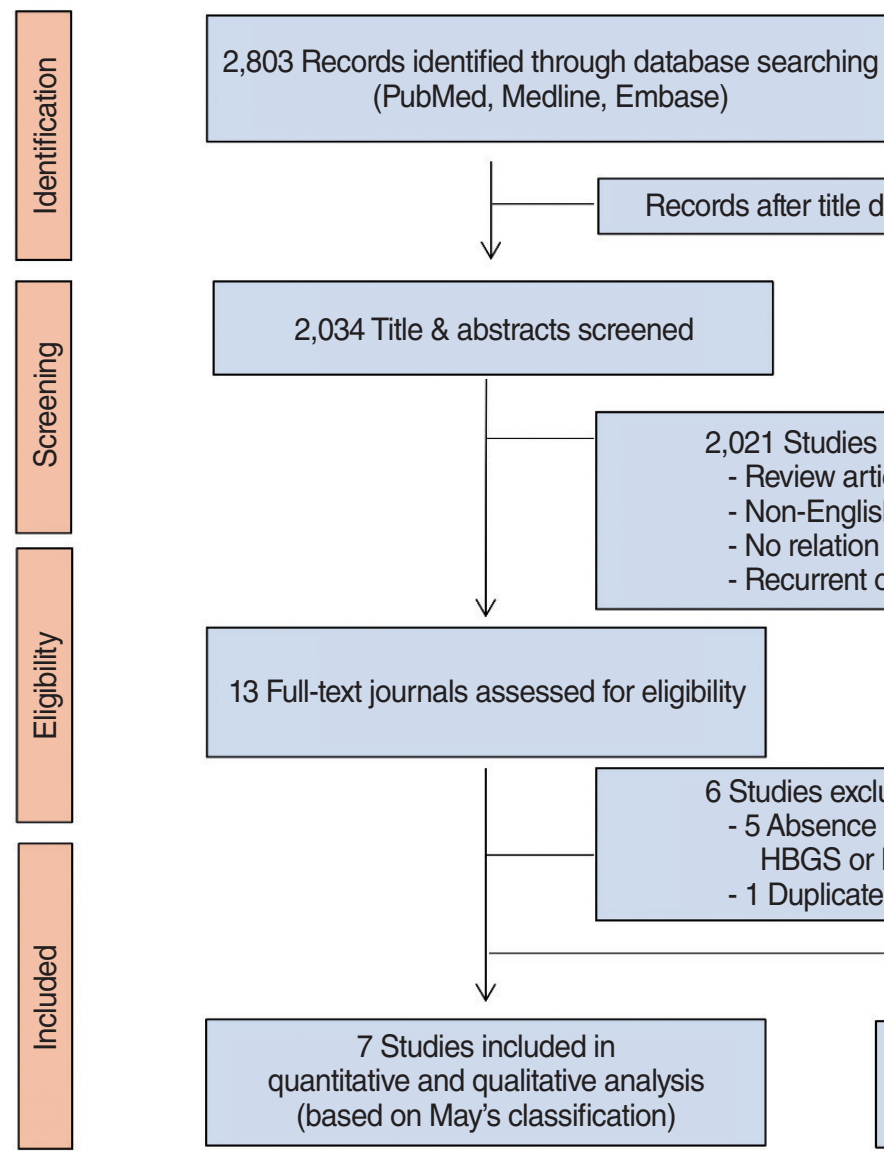

2,034 Title \& abstracts screened

Fig. 1. Preferred Reporting Items for Systematic Reviews and Meta-Analyses (PRISMA) flow diagram outlining the study design. HBGS, HouseBrackmann grading system. 
$<0.10$ were identified, we classified the heterogeneity of the effect size as substantial. For post-hoc analyses, either the trimand-fill method or sensitivity analysis was used to verify the integrity of the quantitative analysis results. Moreover, publication bias was evaluated with a funnel plot if more than three studies were included.

\section{RESULTS}

\section{Characteristics of studies included in the meta-analysis}

The inclusion of studies was determined using a flow diagram (Fig. 1). After identification and screening, 13 studies were assessed for eligibility. As indicated in Table 1, six studies were excluded because they used evaluation methods that were different from the HBGS or May's classification $(n=5)[9,14-17]$ and

Table 1. Characteristics of excluded studies (ordered by study year)

\begin{tabular}{|c|c|}
\hline Study & Reason for exclusion \\
\hline Aoyagi et al. (1988) [14] & $\begin{array}{l}\text { Outcome measures: the degree of facial nerve palsy recovery is evaluated by ENoG and cannot be changed to May's } \\
\text { classification. }\end{array}$ \\
\hline Fisch et al. (1981) [9] & $\begin{array}{l}\text { Outcome measures: the degree of facial nerve palsy recovery is also evaluated by nerve degeneration as ENoG and cannot } \\
\text { be changed to may's classification. }\end{array}$ \\
\hline Adour & $\begin{array}{l}\text { Outcome measures: the degree of facial nerve palsy recovery was evaluated by a unique method of FPRP and FPRI. There } \\
\text { was no change can be evaluated in may's classification. }\end{array}$ \\
\hline Mcneill (1974) [17] & $\begin{array}{l}\text { Outcome measures: the degree of facial nerve paralysis was divided into acceptable and unacceptable; thereby, conversion } \\
\text { to may's classification was impossible. }\end{array}$ \\
\hline Mechelse et al. (1971) [16] & $\begin{array}{l}\text { Outcome measures: the degree of facial nerve palsy recovery was assessed by classifying the frontalis muscle, orbicularis } \\
\text { oculi muscle orbicularis oris muscle from } 0 \text { to } 5 \text { in six stages, so that it could not be replaced with may's classification. }\end{array}$ \\
\hline May et al. (1981) [18] & Subjects: the presumed duplicate cohort, with subsequent studies published by the same author. \\
\hline
\end{tabular}

ENoG, electroneurography; FPRP, facial paralysis recovery profile; FPRI, facial paralysis recovery index.

Table 2. Demographics and clinical characteristics of enrolled studies

\begin{tabular}{|c|c|c|c|c|c|c|c|}
\hline Study/country & Study design & Age (yr, range) & $\begin{array}{l}\text { No. of FND } \\
\text { groups }\end{array}$ & $\begin{array}{c}\text { No. of } \\
\text { controls }\end{array}$ & Surgical indication & $\begin{array}{l}\text { Evaluation } \\
\text { grading }\end{array}$ & Treatment outcome \\
\hline $\begin{array}{l}\text { Li et al. }(2016) / \\
\text { China [19] }\end{array}$ & Quasi-RCT & 21-62 (median, 41.3) & 25 & 13 & $\begin{array}{l}\text { HBGS V or VI CMAP } \\
\text { degeneration }>95 \% \\
\text { (based on EMG) }\end{array}$ & $\begin{array}{l}\text { May's } \\
\text { classification } \\
\text { (modified } \\
\text { HBGS) }\end{array}$ & $\begin{array}{l}\text { Recovery of } \\
\text { facial function, } \\
\text { postoperative } \\
\text { complications }\end{array}$ \\
\hline $\begin{array}{l}\text { Kim et al. (2016)/ } \\
\text { Korea [8] }\end{array}$ & Retrospective & $\begin{array}{l}\text { FND: } 18-76 \text { (mean } \pm S D \\
\text { 48.5 } \pm 17.4) ; \text { control: } 18-74 \\
\text { (mean } \pm S D, 50.4 \pm 16.1)\end{array}$ & 12 & 22 & $\begin{array}{l}\text { Degeneration }>90 \% \\
\text { (based on ENoG) } \\
\text { No voluntery EMG }\end{array}$ & HBGS & $\begin{array}{l}\text { Recovery of facial } \\
\text { function, hearing } \\
\text { threshold }\end{array}$ \\
\hline $\begin{array}{l}\text { Yanagihara et al. } \\
(2001) / J a p a n \\
\text { [20] }\end{array}$ & Retrospective & 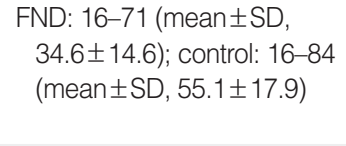 & 58 & 43 & $\begin{array}{l}\text { HBGS V or VI CMAP } \\
\text { degengeration }>95 \% \\
\text { (based on EMG) }\end{array}$ & HBGS & $\begin{array}{l}\text { Recovery of } \\
\text { facial function } \\
\text { Postoperative, } \\
\text { complications }\end{array}$ \\
\hline $\begin{array}{l}\text { Gantz et al. } \\
\text { (1999)/USA, } \\
\text { multicenter [21] }\end{array}$ & Prospective & $\begin{array}{l}\text { FND: 9-58 (mean, 32); } \\
\text { control: 23-66 (mean, 47) }\end{array}$ & 34 & 36 & $\begin{array}{l}\text { Degeneration }>90 \% \\
\text { (based on ENoG) } \\
\text { No voluntary EMG within } \\
2 \text { weeks }\end{array}$ & HBGS & $\begin{array}{l}\text { Recovery of facial } \\
\text { function }\end{array}$ \\
\hline $\begin{array}{l}\text { Gantz et al. } \\
\text { (1999)/USA, } \\
\text { lowa [21] }\end{array}$ & Prospective & $\begin{array}{l}\text { FND: 20-57 (mean, 41); } \\
\text { control: 23-66 (mean, 47) }\end{array}$ & 7 & 11 & $\begin{array}{l}\text { Degeneration }>90 \% \\
\text { (based on ENoG) } \\
\text { No voluntary EMG within } \\
2 \text { weeks }\end{array}$ & HBGS & $\begin{array}{l}\text { Recovery of } \\
\text { facial function, } \\
\text { postoperative } \\
\text { complications }\end{array}$ \\
\hline $\begin{array}{l}\text { May et al. (1985)/ } \\
\text { USA [22] }\end{array}$ & Prospective & NA & 25 & 13 & $\begin{array}{l}\text { Complete paralysis } \\
\text { (and) degeneration } \\
>90 \% \text { (based on } \\
\text { EMG) }\end{array}$ & HBGS & $\begin{array}{l}\text { Recovery of facial } \\
\text { function }\end{array}$ \\
\hline $\begin{array}{r}\text { Brown (1982)/ } \\
\text { Canada [13] }\end{array}$ & Quasi-RCT & NA & 41 & 41 & $\begin{array}{l}\text { Complete paralysis } \\
\text { Unfavorable prognosis } \\
\text { for complete recovery }\end{array}$ & $\begin{array}{l}\text { May's } \\
\text { classification } \\
\text { (modified } \\
\text { HBGS) }\end{array}$ & $\begin{array}{l}\text { Recovery of } \\
\text { facial function, } \\
\text { postoperative } \\
\text { complications }\end{array}$ \\
\hline
\end{tabular}

FND, facial nerve decompression; RCT, randomized controlled trial; HBGS, House-Brackmann grading system; CMAP, compounding muscle action potential; EMG, electromyography; SD, standard deviation; ENoG, electroneurography; NA, not available.

${ }^{a}$ Facial nerve function test showed function of less than $25 \%$ on the affected side. 
Table 3. Comparison of treatment outcomes between FND and conservative treatment

\begin{tabular}{|c|c|c|c|c|c|c|c|c|}
\hline \multirow[b]{2}{*}{ Study/country } & \multirow[b]{2}{*}{ Approach } & \multirow[b]{2}{*}{$\begin{array}{l}\text { Surgical } \\
\text { timing }\end{array}$} & \multirow[b]{2}{*}{$\begin{array}{l}\text { Assessment } \\
\text { timing (mo) }\end{array}$} & \multicolumn{2}{|c|}{ FND } & \multicolumn{2}{|c|}{ Control } & \multirow[b]{2}{*}{$\begin{array}{l}\text { Side effect } \\
\text { (FND) }\end{array}$} \\
\hline & & & & $\begin{array}{l}\text { Recovery of } \\
\text { facial function } \\
\text { HBGS }\end{array}$ & $\begin{array}{c}\text { Recovery of } \\
\text { facial function } \\
\text { May's classification }\end{array}$ & $\begin{array}{c}\text { Recovery of } \\
\text { facial function } \\
\text { HBGS }\end{array}$ & $\begin{array}{c}\text { Recovery of } \\
\text { facial function } \\
\text { May's classification }\end{array}$ & \\
\hline $\begin{array}{l}\text { Li et al. (2016)/ } \\
\text { China [19] }\end{array}$ & $\begin{array}{c}\text { Transmastoid } \\
\text { approach }\end{array}$ & $\begin{array}{l}>2 \text { mo } \\
\quad \text { (delayed) }\end{array}$ & 12 & NA & $\begin{array}{l}\text { (Total }=25) \\
\text { Complete: } 2, \\
\text { fair: } 14 \text {, fail: } 9\end{array}$ & NA & $\begin{array}{l}\text { (Total }=13) \\
\text { Complete: } 1, \\
\text { fair: } 6 \text {, fail: } 6\end{array}$ & $\begin{array}{l}\text { SNHL: 4/25, } \\
\text { tinnitus: } \\
3 / 25\end{array}$ \\
\hline $\begin{array}{l}\text { Kim et al. (2016)/ } \\
\text { Korea [8] }\end{array}$ & $\begin{array}{c}\text { Transmastoid } \\
\text { approach }\end{array}$ & $\begin{array}{l}42 \text { day } \\
(21-70 \\
\text { delayed })\end{array}$ & $6(\mathrm{SD}, 2.5)$ & $\begin{array}{l}\text { (Total =12) } \\
\text { I: 3, II: 6, III: 3, } \\
\text { IV-VI: } 0\end{array}$ & $\begin{array}{l}\text { (Total }=12) \\
\text { Complete: } 3 \text {, } \\
\text { fair: } 9 \text {, fail: } 0\end{array}$ & $\begin{array}{l}\text { (Total }=22) \\
\text { I: } 5, \text { II: } 9, \text { III: 4, } \\
\text { IV: } 3, \mathrm{~V}: 1\end{array}$ & $\begin{array}{l}\text { (Total=22) } \\
\text { Complete: } 5 \text {, } \\
\text { fair: 13, fail: } 4\end{array}$ & $\begin{array}{l}\text { Deterioration } \\
\text { of hearing } \\
\text { threshold } \\
\text { (a mean of } \\
9.7 \mathrm{~dB} \text { ) }\end{array}$ \\
\hline $\begin{array}{l}\text { Yanagihara et al. } \\
\text { (2001)/ } \\
\text { Japan [20] }\end{array}$ & $\begin{array}{c}\text { Transmastoid } \\
\text { approach }\end{array}$ & $\begin{array}{l}>14 \text { day } \\
\quad \text { (delayed) }\end{array}$ & 12 & $\begin{array}{l}\text { (Total }=58) \\
\text { I: } 26, \text { II: 15, } \\
\text { III: 17, IV-VI: } 0\end{array}$ & $\begin{array}{l}\text { (Total }=58) \\
\text { Complete: } 26, \\
\text { fair: } 32 \text {, fail: } 0\end{array}$ & $\begin{array}{l}\text { (Total =43) } \\
\text { I: 10, II: 16, III: } \\
\text { 11, IV-V: } 6\end{array}$ & $\begin{array}{l}\text { (Total }=43) \\
\text { Complete: } 10, \\
\text { fair, } 27 \text {, fail: } 6\end{array}$ & $\begin{array}{c}\text { Transient } \\
\mathrm{CHL}\end{array}$ \\
\hline $\begin{array}{l}\text { Gantz et al. } \\
\text { (1999)/USA, } \\
\text { multicenter [21] }\end{array}$ & MFA & $\begin{array}{r}<14 \text { day } \\
\text { (early) }\end{array}$ & 7 & $\begin{array}{l}\text { (Total = 34) } \\
\text { I: 14, II: 17, III: } \\
\text { 2, IV: 1, V-VI: } 0\end{array}$ & $\begin{array}{l}\text { (Total=34) } \\
\text { Complete: } 14 \text {, } \\
\text { fair: } 19 \text {, fail: } 1\end{array}$ & $\begin{array}{l}\text { (Total =36) } \\
\text { I: 5, II: 10, III: 19, } \\
\text { IV: 2, V-VI: } 0\end{array}$ & $\begin{array}{l}\text { (Total }=36) \\
\text { Complete: } 5 \text {, } \\
\text { fair: } 29 \text {, fail: } 2\end{array}$ & NA \\
\hline $\begin{array}{l}\text { Gantz et al. } \\
\text { (1999)/USA, } \\
\text { lowa [21] }\end{array}$ & MFA & $\begin{array}{l}>14 \text { day } \\
\quad \text { (delayed) }\end{array}$ & 7 & $\begin{array}{l}\text { (Total =7) } \\
\text { I: 0, II: 2, III: 5, } \\
\text { IV-VI: } 0\end{array}$ & $\begin{array}{l}\text { (Total }=7) \\
\text { Complete: } 0 \text {, } \\
\text { fair: } 7 \text {, fail: } 0\end{array}$ & $\begin{array}{l}\text { (Total=11) } \\
\text { I: 0, II: 4, III: 7, } \\
\text { IV-VI: } 0\end{array}$ & $\begin{array}{l}\text { (Total }=11) \\
\text { Complete: } 0, \\
\text { fair: 11, fail: } 0\end{array}$ & $\begin{array}{l}\text { CHL: 1/26, } \\
\text { CSF } \\
\text { leakage: } \\
\text { 1/26 }\end{array}$ \\
\hline $\begin{array}{l}\text { May et al. (1985)/ } \\
\text { USA [22] }\end{array}$ & $\begin{array}{c}\text { Transmastoid } \\
\text { approach }\end{array}$ & $\begin{array}{r}<14 \text { day } \\
\text { (early) }\end{array}$ & $>6$ & $\begin{array}{l}\text { (Total }=25) \\
\text { I: } 0, \text { II: } 5 \\
\text { III: } 11, \text { IV: } 9\end{array}$ & $\begin{array}{l}\text { (Total }=25) \\
\text { Complete: } 0, \\
\text { fair: 16, fail: } 9\end{array}$ & $\begin{array}{l}\text { (Total=13) } \\
\text { I: } 1, \text { II: } 2, \text { III: } 9 \\
\text { IV: } 1\end{array}$ & $\begin{array}{l}\text { (Total=13) } \\
\text { Complete: } 1 \text {, } \\
\text { fair: 11, fail: } 1\end{array}$ & NA \\
\hline $\begin{array}{r}\text { Brown (1982)/ } \\
\text { Canada [13] }\end{array}$ & $\begin{array}{c}\text { Transmastoid } \\
\text { approach }\end{array}$ & $\begin{array}{c}<14 \text { day } \\
\text { (early) }\end{array}$ & $6-12$ & & $\begin{array}{l}\text { (Total }=41) \\
\text { Complete: } 25 \text {, } \\
\text { fair: } 10 \text {, fail: } 6\end{array}$ & & $\begin{array}{l}\text { (Total }=41) \\
\text { Complete: } 20, \\
\text { fair: } 12 \text {, fail: } 9\end{array}$ & $\begin{array}{l}\text { Deafness: } \\
\text { 6/41, } \\
\text { persistent } \\
\text { giddiness: } \\
\text { 2/41 }\end{array}$ \\
\hline
\end{tabular}

FND, facial nerve decompression; HBGS, House-Brackmann grading system; NA, not available; SNHL, sensorineural hearing loss; SD, standard deviation; CHL, conductive hearing loss; MFA, middle fossa approach; CSF, cerebrospinal fluid.

one study was further excluded, including presumed duplicate cohort, with subsequent studies published by the same author [18]. Ultimately, seven studies were selected for quantitative and qualitative analysis [8,13,19-22].

As shown in Table 2, seven studies were included in the metaanalysis: two quasi-randomized controlled trials (RCTs), three prospective and two retrospective case-controlled studies. A total of 381 patients were involved in the meta-analysis; 202 (53.0\%) underwent FND while 179 (47.0\%) underwent conservative treatment (steroid and/or antiviral therapy). The selected studies were conducted between 1982 and 2017. The diagnostic criteria of FND and control group are identical. In detail, all patients enrolled in the meta-analysis were compatible with complete palsy (HBGSV and VI based on HBGS) and/or severe degeneration of facial nerve function (degeneration ratio $>90 \%$ based on electrodiagnostic testing). The surgical approaches for FND were the transmastoid approach $(\mathrm{n}=5)$ and middle fossa approach $(\mathrm{n}=2)$. Additionally, according to the operation timing, early and delayed intervention group were four and three, respectively.

\section{The recovery of facial nerve function}

Table 3 compares the outcomes of each study according to treat- ment approach (FND vs. conservative treatment). All studies contained data on the degree of recovery of facial nerve function. In a pooled analysis (Fig. 2A), based on May's classification, the complete recovery of facial function was significantly higher in the FND group than in the conservative group (OR, 2.06; 95\% CI, 1.22 to $3.48 ; P=0.007)$. Neither heterogeneity nor publication bias was found in studies which reported complete recovery (Fig. 2B). Moreover, sensitivity analysis revealed similar ORs, regardless of a strategy by omitting each study (Fig. 2C).

Meanwhile, the rate of fair recovery (OR, $0.71 ; 95 \%$ CI, 0.42 to $1.21 ; P=0.208$ ) in the FND group was similar to that in the conservative group (Fig. 3A). For failed recovery, no difference in ORs was observed between the two groups (OR, 0.60; 95\% CI, 0.22 to $1.67 ; P=0.327$ ) (Fig. 3B). There was no substantial heterogeneity in studies which reported fair and failed recovery. Moreover, publication bias did not show a noticeable asymmetry on the funnel plot.

Five of eight studies reported the recovery of facial nerve function after using the HBGS. In the pooled analysis (Fig. 4A), $\mathrm{HB}$ scores were improved more in the FND group than in the conservative group (SMD, $-0.30 ; 95 \% \mathrm{CI},-0.82$ to $0.22 ; P=$ 0.26 ), but this improvement did not reach statistical significance. 


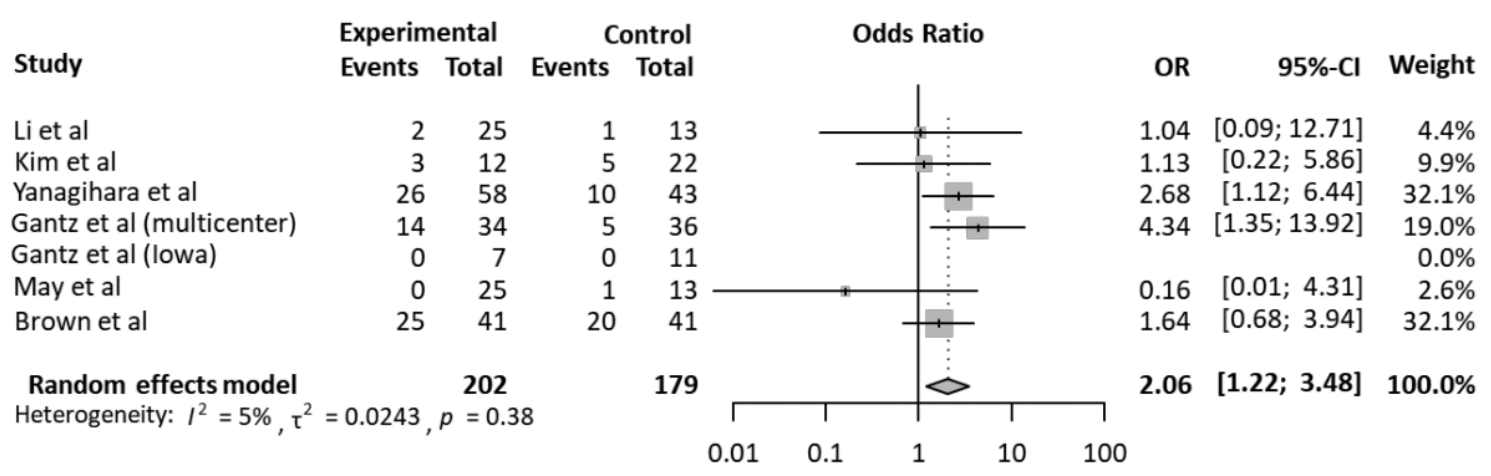

Random-Effects Model

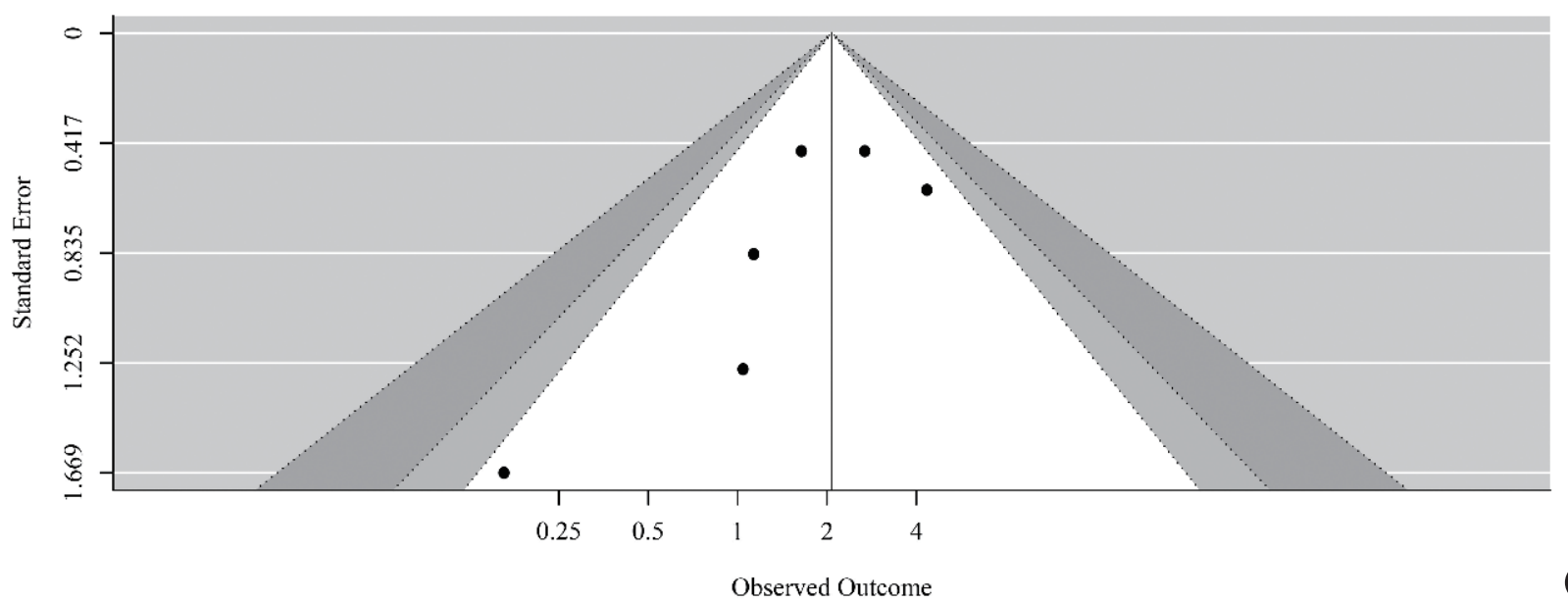

B

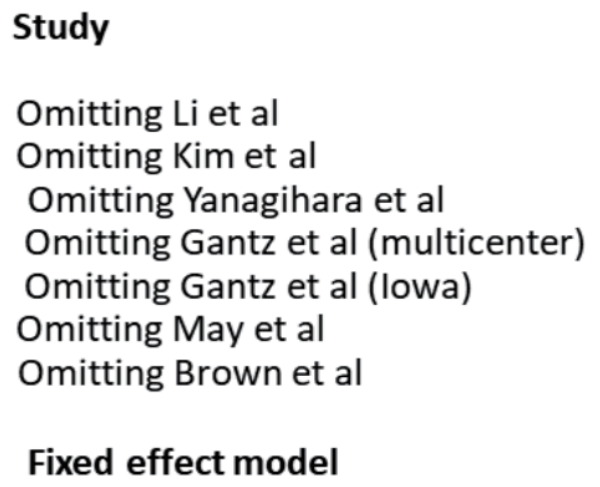

\section{Odds Ratio}

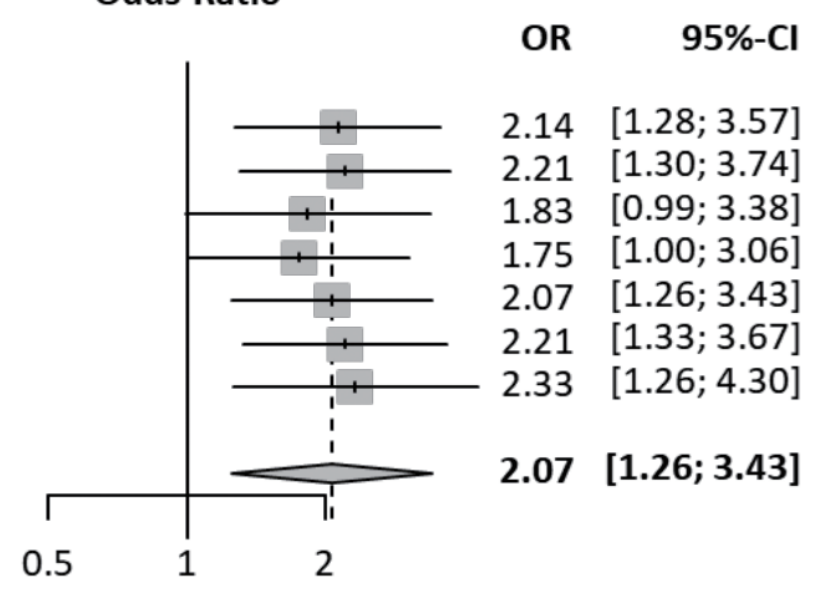

Fig. 2. Comparison of the rate of complete recovery. (A) Forest plot comparing the rate of complete recovery between facial nerve decompression (experimental) and conservative treatment (control) using the odds ratio (OR) and 95\% confidence interval $(\mathrm{Cl})$. Events represent the number of cases with complete recovery based on May's classification. (B) Symmetry based on funnel plot suggesting no publication bias. (C) Sensitivity analysis.

Specifically, a substantial amount of heterogeneity $\left(\mathrm{I}^{2}=72.6 \%\right.$ and $P=0.005$ ) and publication bias was found (Fig. 4B). After adjustment, the trim-and-fill method revealed that the SMD for improvements in HB scores was -0.64 (95\% CI, -1.20 to -0.08 ; $P=0.025)$, indicating significant small-study effects which com- promised the analysis using HBGS.

Subgroup analysis: operation timing

All studies were subjected to subgroup analysis according to the operation timing. Regarding complete recovery, the early inter- 


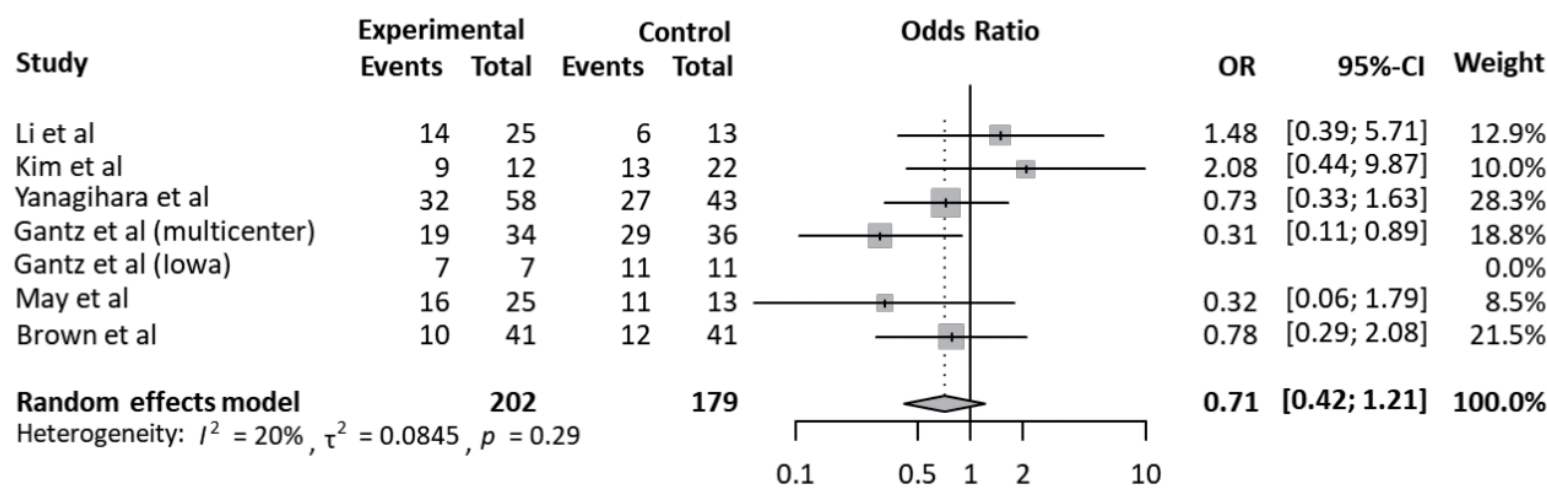

\begin{tabular}{|c|c|c|}
\hline \multirow[b]{2}{*}{ Study } & \multicolumn{2}{|c|}{ Experimental } \\
\hline & Events & Total \\
\hline Li et al & 9 & 25 \\
\hline Kim et al & 0 & 12 \\
\hline Yanagihara et al & 0 & 58 \\
\hline Gantz et al (multicenter) & 1 & 34 \\
\hline Gantz et al (lowa) & 0 & 7 \\
\hline May et al & 9 & 25 \\
\hline Brown et al & 6 & 41 \\
\hline $\begin{array}{l}\text { Random effects model } \\
\text { Heterogeneity: } I^{2}=39 \% \text {, }\end{array}$ & $=0.600$ & $\begin{array}{l}202 \\
p=0\end{array}$ \\
\hline
\end{tabular}

\section{Control Odds Ratio}

nts Tota

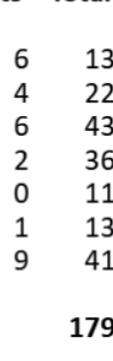

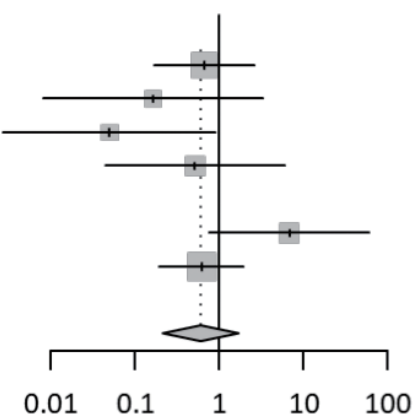

$\begin{array}{rrr}\text { OR } & \text { 95\%-Cl } & \text { Weight } \\ & & \\ 0.66 & {[0.17 ; 2.56]} & 25.0 \% \\ 0.16 & {[0.01 ; 3.33]} & 9.2 \% \\ 0.05 & {[0.00 ; 0.90]} & 9.7 \% \\ 0.52 & {[0.04 ; 5.96]} & 12.6 \% \\ & & 0.0 \% \\ 6.75 & {[0.75 ; 60.76]} & 14.6 \% \\ 0.61 & {[0.20 ; 1.90]} & 28.9 \% \\ & & \\ \mathbf{0 . 6 0} & {[\mathbf{0 . 2 2} \mathbf{1 . 6 7}]} & \mathbf{1 0 0 . 0 \%}\end{array}$

B

Fig. 3. Comparison of the rate of fair and failed recovery. Forest plots comparing the rate of (A) fair recovery and (B) failed recovery between facial nerve decompression (experimental) and conservative treatment (control) using the odds ratio (OR) and 95\% confidence interval (95\% $\mathrm{Cl})$. Events were based on May's classification.
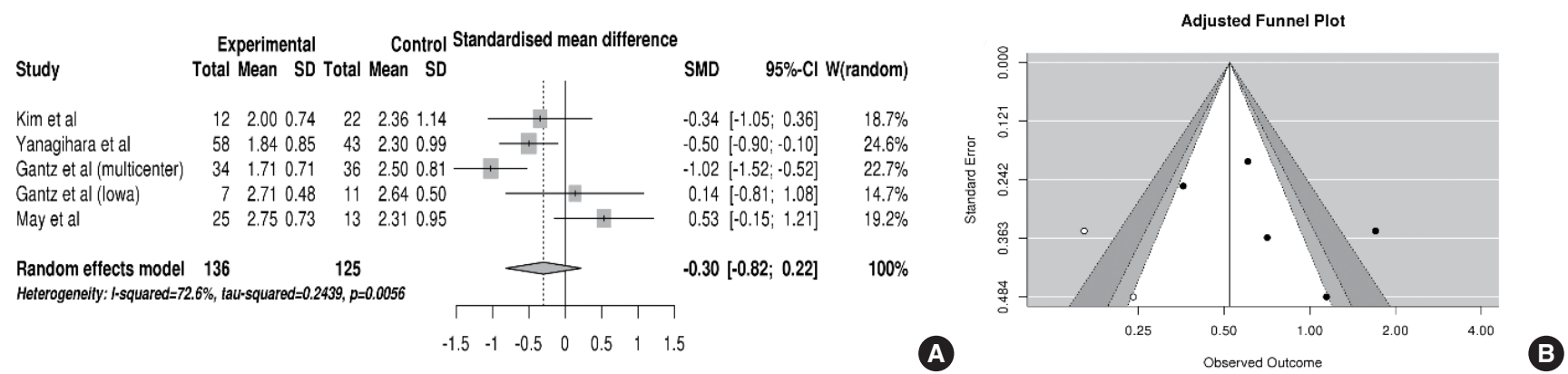

Fig. 4. Comparison of the House-Brackmann grading scores. (A) Forest plot comparing the House-Brackmann grading scores between facial nerve decompression (experimental) and conservative treatment (control) based on the standardized mean difference (SMD). (B) Adjusted publication bias after applying the trim-and-fill method. SD, standard deviation; $\mathrm{Cl}$, confidence interval.

vention group (OR, 2.07; 95\% CI, 0.99 to 4.34) appeared to have a higher OR than the delayed intervention group (OR, 1.90 ; $95 \% \mathrm{CI}, 0.60$ to 6.07$)$, but there was no statistical significance $(P=0.901)$. This tendency was also observed in the early intervention group with regards to fair and failed recovery. Collectively, based on May's classification, there was no significant difference in ORs between the FND and conservative groups, according to operation timing (Fig. 5). For subgroup analysis of the surgical approach, based on May's classification, the degree of recovery of facial nerve function was similar regardless of the surgical approach (Fig. 6).

\section{Postoperative complications}

Data regarding postoperative complications were available in five studies (Table 3). Specifically, hearing loss was evident among patients who underwent FND via transmastoid approach. This hearing loss ranged from transient conductive hearing loss (CHL) to deafness. Among the five studies, the incidence of hearing loss was $12.0 \%$ (11/91). In patients who underwent FND via the middle fossa approach, CHL and cerebro- 


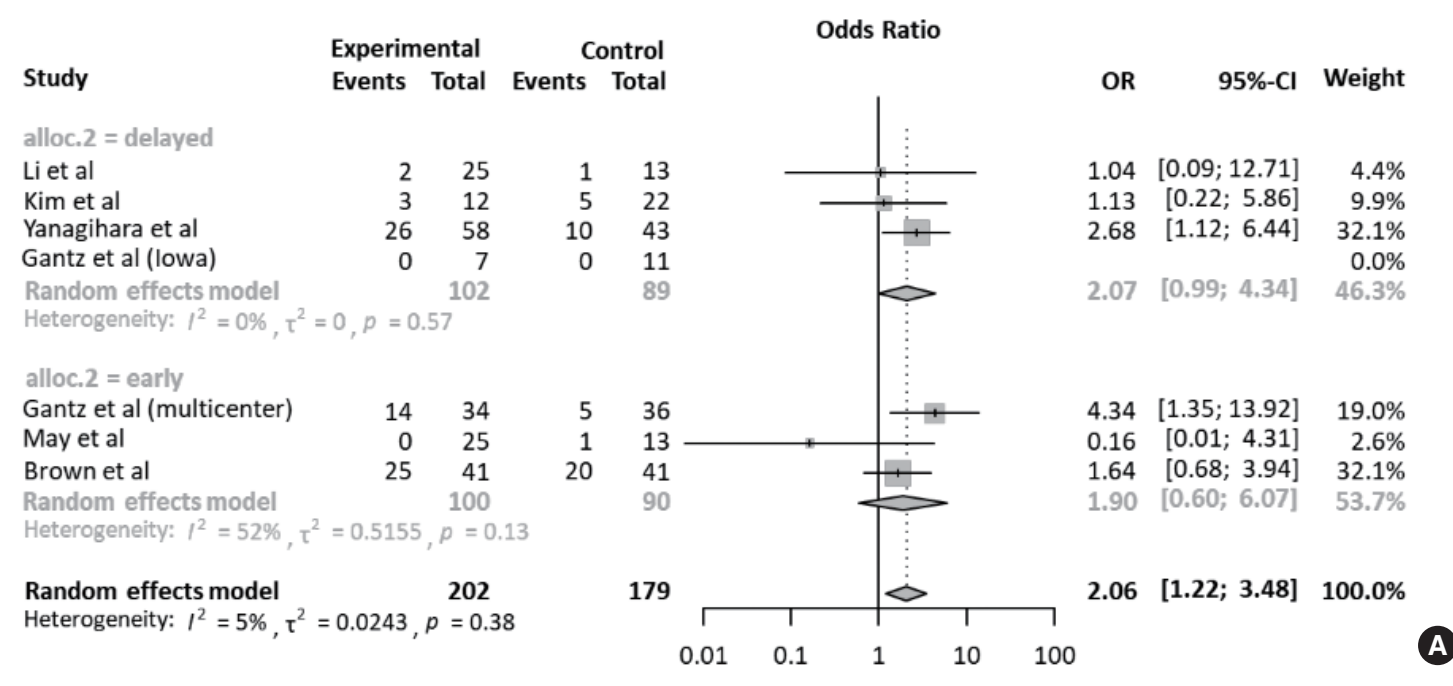

$\begin{array}{lll} & & \\ \text { Experimental } & \text { Control } & \text { Odds Ratio } \\ \text { Study } & \text { Events Total Events Total }\end{array}$

alloc.2 = delayed

Li et al

Kim et al

Yanagihara et al

Random effects model

Heterogeneity: $t^{2}=0 \%, \tau^{2}=0, p=0.41$

alloc.2 = early

Gantz et al (multicenter)

May et al

Brown et al

Random effects model

Heterogeneity: $L^{2}=0 \%, \tau^{2}=0, P=0.40^{-100}$

Random effects model

Heterogeneity: $l^{2}=20 \%, \tau^{2}=0.0845, p=0.29$
Gantz et al (lowa)

13
22
43
11
89
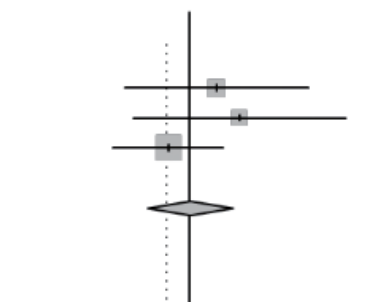

OR $\quad 95 \%-\mathrm{Cl}$ Weight

$1.48 \quad[0.39 ; 5.71] \quad 12.9 \%$

$2.08 \quad[0.44 ; 9.87] \quad 10.0 \%$

$0.73[0.33 ; 1.63] \quad 28.3 \%$

$1.01[0.54 ; 1.91] \quad \begin{array}{r}0.0 \% \\ 51.2 \%\end{array}$

$0.31 \quad[0.11 ; 0.89] \quad 18.8 \%$

$0.32[0.06 ; 1.79] \quad 8.5 \%$

$0.78 \quad[0.29 ; 2.08] \quad 21.5 \%$

$0.47[0.24 ; 0.92] \quad 48.8 \%$

$0.71[0.42 ; 1.21] 100.0 \%$

179

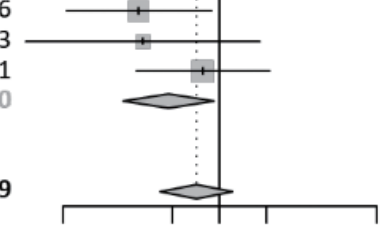

$\begin{array}{lllll}0.1 & 0.5 & 1 & 2 & 10\end{array}$

B

OR $\quad 95 \%-\mathrm{Cl}$ Weight

$0.66 \quad[0.17 ; 2.56] \quad 25.0 \%$

$0.16 \quad[0.01 ; 3.33] \quad 9.2 \%$

$0.05 \quad[0.00 ; 0.90] \quad 9.7 \%$

$0.28[0.06 ; 1.29] \quad \begin{array}{r}0.0 \% \\ 43.9 \%\end{array}$

Gantz et al (lowa)

Random effects model

Experimental Control

Events Total Events Total

alloc. 2 = delayed

Li et al

Kim et al

925

$\begin{array}{ll}6 & 13 \\ 4 & 22 \\ 6 & 43 \\ 0 & 11 \\ & 89\end{array}$

Heterogeneity: $1^{2}=29 \%, \tau^{2}=0.5897, p=0.25$

89

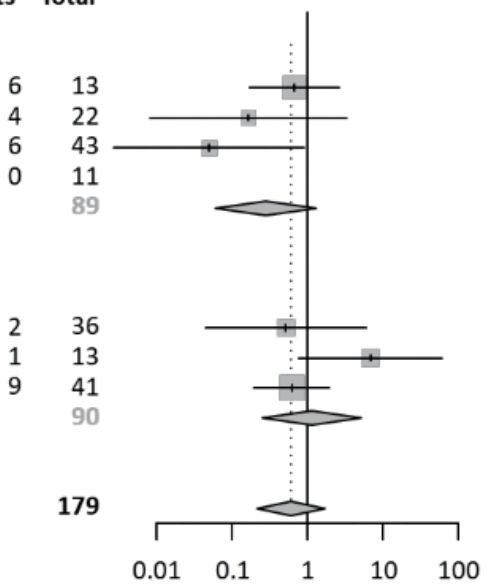

alloc.2 = early

Gantz et al (multicenter)

May et al

Brown et al

Random effects model

$\begin{array}{rr}1 & 34 \\ 9 & 25 \\ 6 & 41 \\ & 100\end{array}$

Heterogeneity: $I^{2}=48 \%, \tau^{2}=0.8665, p=0.14$

Random effects model

202

Heterogeneity: $l^{2}=39 \%, \tau^{2}=0.6001, p=0.14$

$0.52 \quad[0.04 ; 5.96] \quad 12.6 \%$

$6.75[0.75 ; 60.76] \quad 14.6 \%$

$0.61 \quad[0.20 ; 1.90] \quad 28.9 \%$

$1.13[0.25 ; 5.07] \quad 56.1 \%$

$0.60[0.22 ; 1.67] \quad 100.0 \%$ 


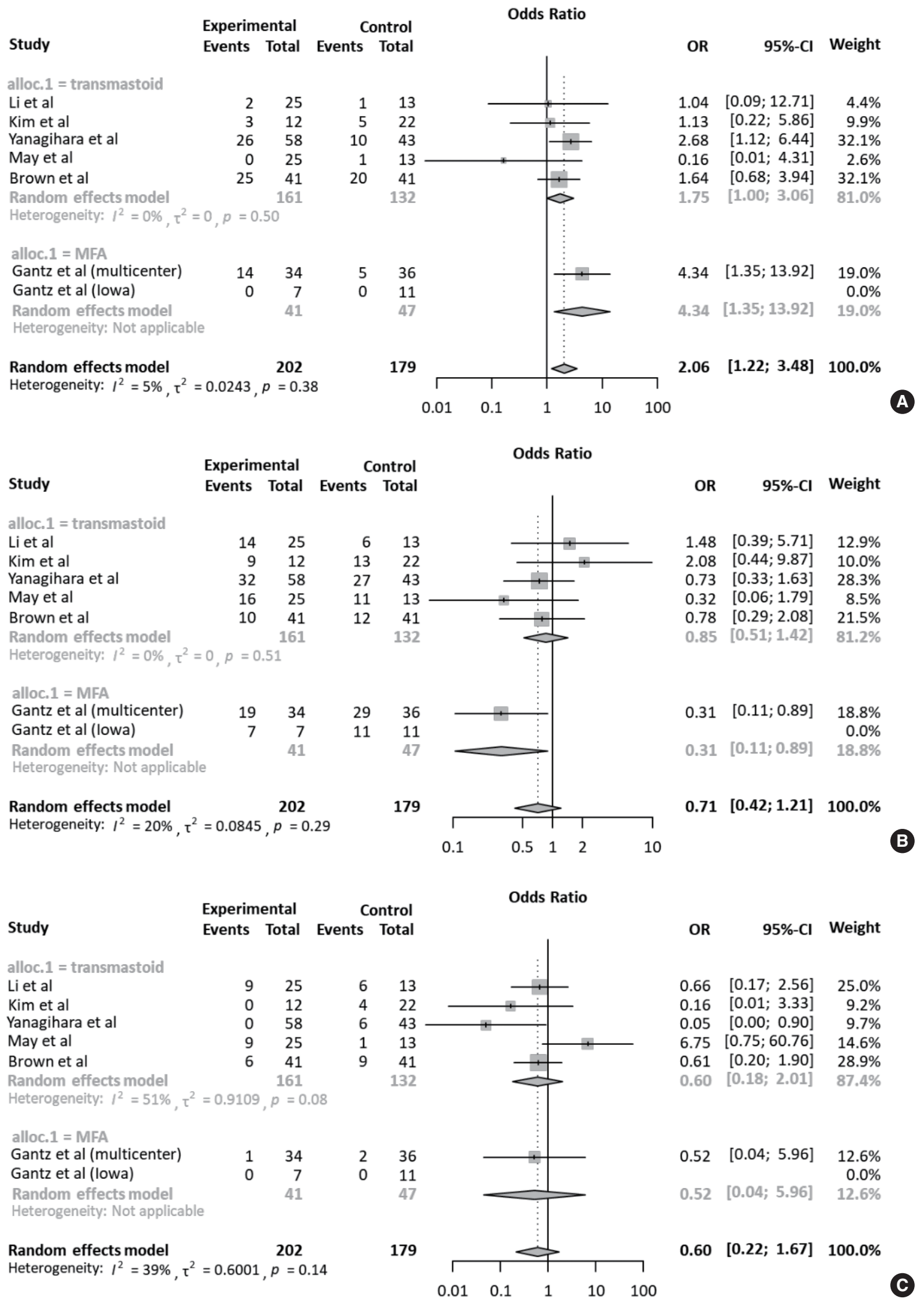

Fig. 6. Subgroup analyses according to surgical approach. Forest plot comparing the recovery of facial nerve function according to the surgical approach (transmastoid approach vs. middle fossa approach). (A) Complete recovery. (B) Fair recovery. (C) Failed recovery. OR, odds ratio; $\mathrm{Cl}$, confidence interval. 
spinal fluid leakage were observed. Due to the lack of information on complications in studies involving conventional treatment, statistical analysis was not possible.

\section{Quality assessment and publication bias}

We assessed the risk of bias in quasi-RCTs based on the Cochrane Collaboration tool (Supplementary Table 2). Additionally, we used the Newcastle-Ottawa Scale criteria to examine the internal validity of prospective and retrospective studies (Supplementary Table 3).

\section{DISCUSSION}

We compared the therapeutic efficacy of FND with conservative treatment through a systematic review and meta-analysis based on seven eligible studies. To the best of our knowledge, our quantitative analyses were based on the largest number of studies, providing meticulous interpretations of integrity regarding potential bias. Interestingly, FND significantly enhanced the rate of complete recovery compared to conventional treatment. Although different prognostic perspectives on surgical approach and operation timing have been suggested, our subgroup analyses did not show significant differences.

Bell's palsy, the most common cause of acute FNP, is considered idiopathic [23]. Although various mechanisms, including viral infections, vascular ischemia, and immune-mediated processes, have been suggested to contribute to Bell's palsy [24,25], the exact pathophysiology of Bell's palsy has not yet been clearly elucidated. In anatomical perspectives, the diameter of the meatal segment of the facial nerve is small (approximately $0.68 \mathrm{~mm}$ ) at the point where it enters the fallopian canal and is susceptible to inflammation and edema [26]. Thus, the edematous swelling of the fallopian canal which underlies Bell's palsy decreases the room for expansion in a rigid bony canal [27], potentially leading to severe nerve damage and even necrosis and fibrosis. More than $90 \%$ nerve degeneration within 14 days after the onset of Bell's palsy is correlated with poor prognosis [28], indicating the need for FND. Opening the bony canal and subsequently releasing the pressure on the nerve sheath causes decompression of the nerve fibers, which can improve the circulation and minimize damage to distal nerve fibers $[29,30]$. Thus, FND prevents continuous nerve degeneration and restores facial nerve function.

However, the therapeutic role of FND in complete Bell's palsy remains unclear. Although evidence on the therapeutic efficacy of FND in improving facial nerve function has increased since the Cochrane review, a recent meta-analysis reported no difference in HBGS between surgical and medical treatment. The study was limited by a significant higher of heterogeneity. Moreover, our current study revealed that the meta-analysis using HBGS was biased as documented by the trim-and-fill method. Based on this, we adopted the May's classification, namely a modified HBGS, to enhance the statistical power and integrity. As shown in Figs. 2 and 3, Neither heterogeneity nor publication bias was found. In other words, the May's classification in this study provides a more reliable interpretation of meta-analysis than HBGS. However, in prognosis perspective to distinguish subtle changes, there seems to be no difference between two evaluation methods when considering the May's classification is based upon HBGS. Our results indicate that FND is closely associated with complete recovery (HBGS I). This information could be clinically significant considering that $10 \%$ of patients with complete palsy only partially recover despite combination therapy with prednisolone and valganciclovir [7].

Several studies reported improved outcomes following early intervention (within 2 weeks after onset) compared to delayed intervention (more than 2 weeks after onset) [21]. A recent meta-analysis demonstrated that middle fossa decompression within 14 days of symptom onset improved the HBGS compared to medical management [11]. Early intervention has been shown to ameliorate the risk of ischemia in the fallopian canal, which is correlated with prognosis [13]. Given that Wallerian degeneration originates from long-standing compression of the facial nerve in the fallopian canal [31], delayed intervention is likely to be associated with poor prognosis. Nonetheless, evidence on benefits of delayed decompression surgery which occurs within 90 days after onset has increased for patients who cannot afford early surgery [32]. Also, our results indicated that the therapeutic efficacy of FND became more slightly evident if those with complete Bell's palsy underwent FND less than 14 days from the onset of symptoms, but with no statistical significance. Collectively, early intervention may lead to better outcomes, but the window of the exact operation timing to rescue the benefit from FND remains controversies.

In light of the presumed anatomical etiology of Bell's palsy, the middle cranial fossa approach which can be used to identify and decompress the proximal labyrinthine segment of the facial nerve may be feasible compared to the transmastoid approach $[11,21]$. Indeed, a previous study which employed the transmastoid approach showed no significant benefit of this approach to complete facial palsy [13], suggesting that the meatal foramen may be key to the pathogenesis of Bell's palsy. However, we did not find any difference in treatment outcomes based on surgical approach. This may be attributed to the inclusion of recent studies which showed that using the transmastoid approach resulted in either complete or fair recovery in most cases. Additionally, it remains unknown whether the surgical approach is an independent prognostic factor when considering clinical factors such as age, comorbidities, and initial status of facial nerve function $[33,34]$. Therefore, a surgical approach may not be an independent determinant of the prognosis after adjusting for possible confounders.

By comparing the therapeutic efficacy of FND with conservative treatment in in patients with complete Bell's palsy following 
medical treatment, our results provide insights on appropriate determination of treatment strategy and timely intervention to restore the facial nerve function. Nevertheless, there are some limitations that should be addressed in future studies. First, even though our results were based on the largest number of studies, small number of randomized controlled studies limit the integrity of the analysis. Moreover, most patients in this study underwent medical treatment before undergoing FND. To be precise, the present study made a direct comparison of facial function recovery between FND following medical treatment and only medical treatment followed by observation, except for one study [13]. In other words, the therapeutic effects of steroid or antiviral therapy were not controlled in the FND group. Thus, randomized controlled studies involving a larger cohort are warranted to further support our current conclusions. Second, some of the selected studies had potential biases, as documented by the Newcastle-Ottawa Scale (Supplementary Table 2), albeit with symmetry in the funnel plot. Given the bias, evaluation with May's classification seems to be more reliable for verifying the therapeutic role of FND than evaluation with HBGS, as shown in Fig. 5. Third, neither HBGS nor May's classification can differentiate subtle changes in facial function, as well as limitations of interobserver agreement. These concerns have led to the proposal of additional systems, such as deep-learning based approach to measuring the precise facial function [35]. Lastly, risk factors that affect the recovery of facial nerve function, such as age and comorbidities (i.e., hypertension and diabetes), were not considered in our selected studies [36].

Taken together, FND can be a possible treatment option in patients with complete Bell's palsy, especially for complete recovery. In subgroup analyses, the recovery of facial nerve function was similar regardless of the surgical approach.Additionally, early intervention with FND within 14 days from the onset of Bell's palsy seems to increase the therapeutic efficacy, but the optimal time window remains unclear. Our results may be useful for decision-making and outcome prediction in patients with complete Bell's palsy. However, FND should be determined carefully when considering the risk of bias and possible complications.

\section{CONFLICT OF INTEREST}

No potential conflict of interest relevant to this article was reported.

\section{ACKNOWLEDGMENTS}

This study was supported by a clinical research grant from the SMG-SNU Boramae Medical Center, Seoul, Korea.

\section{ORCID}

Sang-Yeon Lee https://orcid.org/0000-0003-3566-8708

Jeon Seong https://orcid.org/0000-0003-3644-0289

Young Ho Kim https://orcid.org/0000-0001-7328-5068

\section{AUTHOR CONTRIBUTIONS}

Conceptualization, Data curation, \& Formal analysis: SYL. Funding acquisition: YHK. Methodology, Project administration, Visualization, \& Writing - original draft: SYL. Writing - review \& editing: all authors.

\section{SUPPLEMENTARY MATERIALS}

Supplementary materials can be found via https://doi.org/10. 21053/ceo.2019.00535.

\section{REFERENCES}

1. Sun DQ, Andresen NS, Gantz BJ. Surgical management of acute facial palsy. Otolaryngol Clin North Am. 2018 Dec;51(6):1077-92.

2. Peitersen E. Natural history of Bell's palsy. Acta Otolaryngol Suppl. 1992;492:122-4.

3. Andresen NS, Sun DQ, Hansen MR. Facial nerve decompression. Curr Opin Otolaryngol Head Neck Surg. 2018 Oct;26(5):280-5.

4. Mantsopoulos K, Psillas G, Psychogios G, Brase C, Iro H, Constantinidis J. Predicting the long-term outcome after idiopathic facial nerve paralysis. Otol Neurotol. 2011 Jul;32(5):848-51.

5. Holland NJ, Weiner GM. Recent developments in Bell's palsy. BMJ. 2004 Sep;329(7465):553-7.

6. Adour KK. Combination treatment with acyclovir and prednisone for Bell palsy. Arch Otolaryngol Head Neck Surg. 1998 Jul;124(7): 824.

7. Peitersen E. Bell's palsy: the spontaneous course of 2,500 peripheral facial nerve palsies of different etiologies. Acta Otolaryngol Suppl. 2002;(549):4-30.

8. Kim SH, Jung J, Lee JH, Byun JY, Park MS, Yeo SG. Delayed facial nerve decompression for Bell's palsy. Eur Arch Otorhinolaryngol. 2016 Jul;273(7):1755-60.

9. Fisch U. Surgery for Bell's palsy. Arch Otolaryngol. 1981 Jan;107(1): $1-11$.

10. McAllister K, Walker D, Donnan PT, Swan I. Surgical interventions for the early management of Bell's palsy. Cochrane Database Syst Rev. 2013 Oct;(10):CD007468.

11. Casazza GC, Schwartz SR, Gurgel RK. Systematic review of facial nerve outcomes after middle fossa decompression and transmastoid decompression for Bell's palsy with complete facial paralysis. Otol Neurotol. 2018 Dec;39(10):1311-8.

12. Liberati A,Altman DG, Tetzlaff J, Mulrow C, Gotzsche PC, Ioannidis JP, et al. The PRISMA statement for reporting systematic reviews and meta-analyses of studies that evaluate health care interventions: explanation and elaboration. PLoS Med. 2009 Jul;6(7):e1000100.

13. Brown JS. Bell's palsy: a 5 year review of 174 consecutive cases: an attempted double blind study. Laryngoscope. 1982 Dec;92(12): 
1369-73.

14. Aoyagi M, Koike Y, Ichige A. Results of facial nerve decompression. Acta Otolaryngol Suppl. 1988;446:101-5.

15. Adour KK, Byl FM, Hilsinger RL Jr, Kahn ZM, Sheldon MI.The true nature of Bell's palsy: analysis of 1,000 consecutive patients. Laryngoscope. 1978 May;88(5):787-801.

16. Mechelse K, Goor G, Huizing EH, Hammelburg E, van Bolhuis AH, Staal A, et al. Bell's palsy: prognostic criteria and evaluation of surgical decompression. Lancet. 1971 Jul;2(7715):57-9.

17. McNeill R. Facial nerve decompression. J Laryngol Otol. 1974 May; 88(5):445-55.

18. May M, Blumenthal F, Taylor FH. Bell's palsy: surgery based upon prognostic indicators and results. Laryngoscope. 1981 Dec;91(12): 2092-103.

19. Li Y, Sheng Y, Feng GD, Wu HY, Gao ZQ. Delayed surgical management is not effective for severe Bell's palsy after two months of onset. Int J Neurosci. 2016 Nov;126(11):989-95.

20. Yanagihara N, Hato N, Murakami S, Honda N. Transmastoid decompression as a treatment of Bell palsy. Otolaryngol Head Neck Surg. 2001 Mar;124(3):282-6.

21. Gantz BJ, Rubinstein JT, Gidley P, Woodworth GG. Surgical management of Bell's palsy. Laryngoscope. 1999 Aug;109(8):1177-88.

22. May M, Klein SR, Taylor FH. Idiopathic (Bell's) facial palsy: natural history defies steroid or surgical treatment. Laryngoscope. 1985 Apr; 95(4):406-9.

23. Mattox DE. Clinical disorders of the facial nerve. In: Flint PW, editor. Otolaryngology head and neck surgery review. 3rd ed. St. Louis (MO): Mosby-Year Book; 1998. p. 2767-84.

24. Greco A, Gallo A, Fusconi M, Marinelli C, Macri GF, deVincentiis M. Bell's palsy and autoimmunity. Autoimmun Rev. 2012 Dec;12(2): 323-8.

25. Paolino E, Granieri E, Tola MR, Panarelli MA, Carreras M. Predisposing factors in Bell's palsy: a case-control study. J Neurol. 1985;
232(6):363-5

26. Jain S, Kumar S. Bell's palsy: a need for paradigm shift? Ann Otol Neurotol. 2018;1(01):034-9.

27. Owusu JA, Boahene KD. Bell's palsy. In: Kountakis SE, editor. Encyclopedia of otolaryngology, head and neck surgery. Berlin, Heidelberg: Springer; 2013. p. 256-8.

28. Baugh RF, Basura GJ, Ishii LE, Schwartz SR, Drumheller CM, Burkholder R, et al. Clinical practice guideline: Bell's palsy. Otolaryngol Head Neck Surg. 2013 Nov;149(3 Suppl):S1-27.

29. Pulec JL. Early decompression of the facial nerve in Bell's palsy. Ann Otol Rhinol Laryngol. 1981 Nov-Dec;90(6 Pt 1):570-7.

30. Gelberman RH, Eaton RG, Urbaniak JR. Peripheral nerve compression. Instr Course Lect. 1994;43:31-53.

31. Jackson CG, von Doersten PG. The facial nerve: current trends in diagnosis, treatment, and rehabilitation. Med Clin North Am. 1999 Jan;83(1):179-95.

32. Berania I, Awad M, Saliba I, Dufour JJ, Nader ME. Delayed facial nerve decompression for severe refractory cases of Bell's palsy: a 25-year experience. J Otolaryngol Head Neck Surg. 2018 Jan; 47(1):1.

33. Gillman GS, Schaitkin BM, May M, Klein SR. Bell's palsy in pregnancy: a study of recovery outcomes. Otolaryngol Head Neck Surg. 2002 Jan;126(1):26-30.

34. Lee DH, Chae SY, Park YS, Yeo SW. Prognostic value of electroneurography in Bell's palsy and Ramsay-Hunt's syndrome. Clin Otolaryngol. 2006 Apr;31(2):144-8.

35. Brenner MJ, Neely JG. Approaches to grading facial nerve function. Semin Plast Surg. 2004 Feb;18(1):13-22.

36. Takemoto N, Horii A, Sakata Y, Inohara H. Prognostic factors of peripheral facial palsy: multivariate analysis followed by receiver operating characteristic and Kaplan-Meier analyses. Otol Neurotol. 2011 Aug;32(6):1031-6. 